\title{
SPECTROSCOPIC OBSERVATIONS OF DIFFERENTIAL ROTATION WITH HEIGHT IN THE SOLAR ENVELOPE*
}

\author{
W. C. LIVINGSTON \\ Kitt Peak National Observatory ${ }^{\dagger}$, Tucson, Ariz., U.S.A.
}

\begin{abstract}
The problem of determining the spectroscopic rotation of the sun is not one of instrumental sensitivity, which can be about $10 \mathrm{~m} / \mathrm{sec}$, but rather of abstracting the rotational component from the hierarchy of long-lived surface currents. Required are continued daily observations over long periods of time and this task is not presently feasible at most observatories. Past observations have indicated a height gradient of rotational angular velocities. We have determined the photosphere and chromosphere angular velocity gradient from observations across the disk of $C 5380 \AA(h \simeq-250 \mathrm{~km})$, $\mathrm{CN} 3882 \AA(h \simeq 0 \mathrm{~km}), \mathrm{Ca}^{+} \mathrm{K}_{3}(h \simeq+5000 \mathrm{~km})$, together with other Fraunhofer lines of intermediate origin while $\mathrm{K}$-line spectra of prominences are used to sense the chromosphere-corona interface.

Preliminary results for the disk measurements indicate a constant rotation through the photosphere, but an increase through the chromosphere up to $20-30 \%$ for the $\mathrm{Ca}^{+} \mathrm{K}_{3}$ layers.
\end{abstract}

\section{The Observational Problem}

The task of measuring the rotation of the sun, as compared with a star, is simplified by the enormous available light and the fact that we deal with a resolved disk. High light intensity permits a spectroscopic Doppler sensitivity of about $10 \mathrm{~m} / \mathrm{sec}$ for a Fraunhofer line with an equivalent width of about $100 \mathrm{~m} \AA$. The resolved disk allows us, in principle, to directly measure the motion of rotation. Unfortunately, this

TABLE I

Approximate sizes, lifetimes and velocities of convective disturbances at the solar surface

\begin{tabular}{|c|c|c|c|c|}
\hline & \multirow[t]{2}{*}{$s(\mathrm{~km})$} & \multirow[t]{2}{*}{$\tau$} & \multicolumn{2}{|c|}{$V(\mathrm{~km} / \mathrm{sec}$ p. to p. $)$} \\
\hline & & & Photo & Chrom. \\
\hline ('White-light') granulation & $\simeq 10^{3}$ & $\simeq 7^{\mathrm{m}}$ & $1-2$ & \\
\hline Oscillatory elements & $2 \times 10^{3}$ & $5^{\mathrm{m}}$ & 0.5 & $\begin{array}{l}4 \\
\left(\mathrm{Ca}+\mathrm{K}_{3}\right)\end{array}$ \\
\hline Supergranulation & $30 \times 10^{3}$ & $1^{\mathrm{d}}$ & 0.5 & \\
\hline 'Giant granules' & $300 \times 10^{3} ?$ & $>1^{\mathrm{d}} ?$ & $0.2 ?$ & \\
\hline
\end{tabular}

rotational aspect is somewhat masked by the hierarchy of velocity fields found on the solar surface. Some properties of these currents - in order of increasing spatial extent are summarized in Table $\mathrm{I}$.

The confusion of the fine granules and photospheric oscillatory fields can be made

* Kitt Peak National Observatory Contribution No. 53I.

$\dagger$ Operated by The Association of Universities for Research in Astronomy, Inc., under contract with the National Science Foundation. 
negligible by spatial averaging at the telescope - using an out-of-focus image or an integrating lens. However, a reduction of the chromospheric oscillations (because of their magnitude) and the supergranular and hypothetical 'giant granules' (because of their long life and spatial extent) requires the averaging of observations over many days and even weeks. So far, limitations in telescope time have prevented us from making such an average and thus determining fully the rotation of the sun.

What one can do with limited observational material is to study how the angular velocity of rotation varies with height through the solar envelope. Past observations have indicated a surprising increase in the rotational velocity of the sun for the higher layers. Certain Fraunhofer lines, suitably chosen because of their height of formation, permit a sensing of velocities at different layers in the photosphere and chromosphere. Further out, velocities in the chromosphere-corona interface can be obtained from a statistical analysis of prominence radial velocities. A program of these measurements is being pursued - although many observations are needed because of the large random motions of the individual prominences.

\section{The Disk Observations}

All disk observations are made using the 'Doppler compensator' of the solar magnetograph. This magnetograph is an accessory to the $13.7 \mathrm{~m}$ vertical spectrograph which is normally fed by the main $80-\mathrm{cm}$ image. At the focus of the spectrograph the position of the Fraunhofer line is sensed by a double slit arrangement, the light from either wing of the line being directed to photomultipliers (Figure 1). The diferunce signal from these photomultipliers turns a torque motor, connected to the parallel glass plate, until the line is centered and the signal becomes zero. The position angle of this plate is sensed by a coaxial microsyn generator whose output is recorded on a strin chart. A knowledge of the spectrograph dispersion, the index of refraction and thiciness of the tipping plate, together with the angle-display sensitivity of the microsvn. suffice to reduce any line shift to change of wavelength and hence relative velocity change. The r.m.s. system noise is about $10 \mathrm{~m} / \mathrm{sec}$ with a $10 \mathrm{~Hz}$ bandwidth. This nowse is completely negligible compared with the above mentioned solar effects. (There are actually two separate systems so that two lines can be studied simultaneously.)

The solar image is scanned across the spectrograph entrance slit at a constant rate and at a constant heliographic latitude. Figure 2 indicates a typical photospheric velocity and brightness recording. A straight line fit by eye is made to the velocity trace giving most weight to undisturbed regions, and ignoring certain limb effects. The slope of this line, reduced to angular rotation (sidereal, deg/day) becomes one data point for subsequent averaging. The equatorial rates reported here consist of a straight average of eight double scans, i.e. across and return, in two degree latitude increments. Thus a mean over $\pm 8^{\circ}$ latitude is obtained.

A list of the lines used, how the exit slits are positioned with respect to the line profile and the estimated height of formation of that part of the line being analysec. is given in Table II. Note that, except for the $\mathrm{Ca}^{+} \mathrm{K}_{3}$ line, the core of the line is noI 


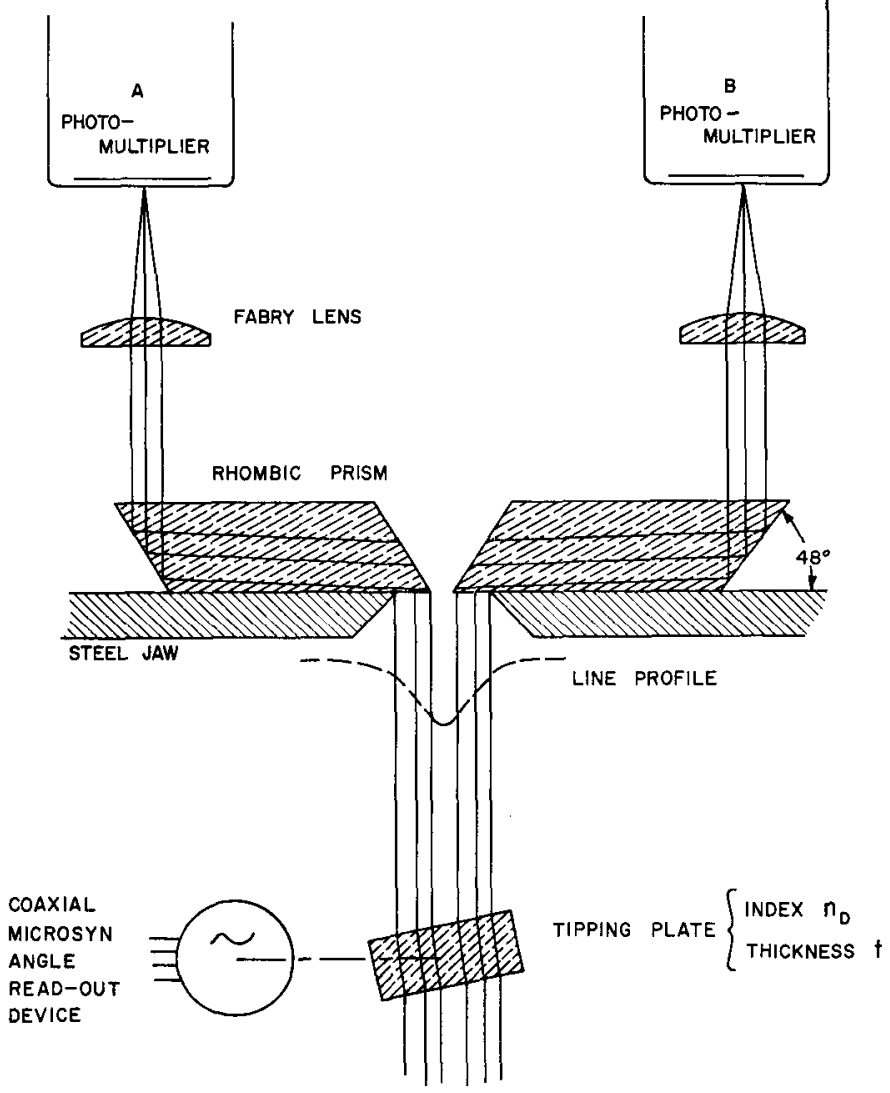

Fig. 1. Arrangement at the focus of the spectrograph for measurement of relative Fraunhofer line displacement. The system exists in duplicate, permitting the simultaneous recording of two lines.

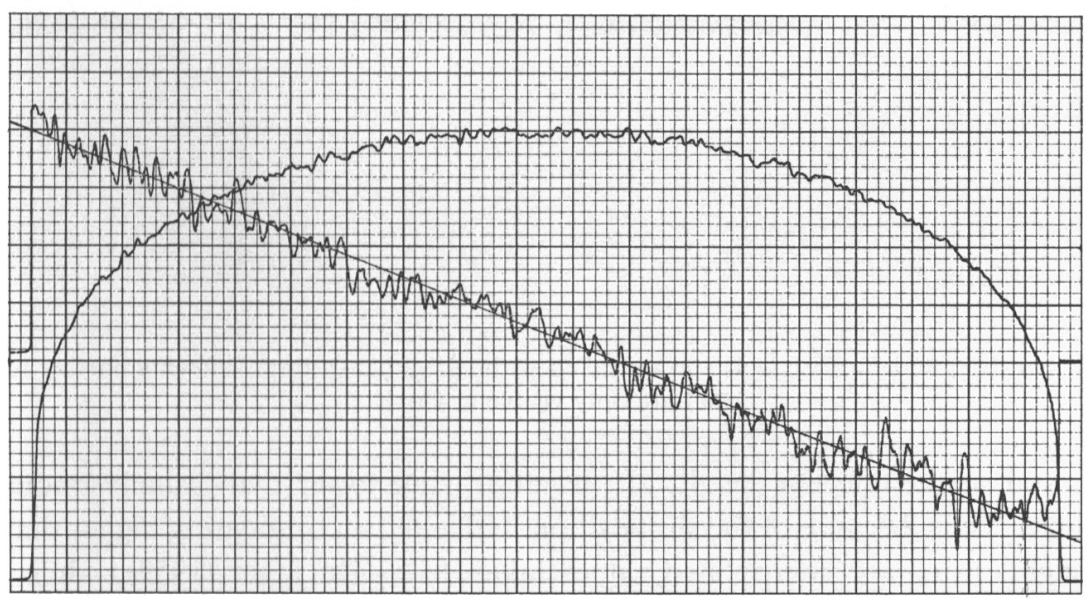

Fig. 2. Typical superposed line displacement (velocity) and brightness records for a single scan across the heliographic equator. A linear fit is made to the velocity record. 


\section{TABLE II}

Equivalent width, excitation potential, double slit widths and separations, and estimated height of formation for lines used in this study

\begin{tabular}{lclll} 
& $\begin{array}{l}w \\
(\mathrm{~m} \AA)\end{array}$ & $\begin{array}{l}\text { e.p. } \\
\text { (volts) }\end{array}$ & $\begin{array}{l}\text { Slits } \\
(\AA)\end{array}$ & $\begin{array}{l}h \\
(\mathrm{~km})\end{array}$ \\
\hline $\mathrm{C} 5380.3$ & 26 & 7.68 & $0.03-0.08-0.03$ & -250 \\
$\mathrm{Fe} 3891.9$ & 88 & 3.41 & $0.02-0.05-0.02$ & \\
$\mathrm{Fe} 3944.9$ & 72 & 2.99 & $0.02-0.05-0.02$ & \\
$\mathrm{Fe} 4842.7$ & 44 & 4.22 & $0.03-0.09-0.03$ & -150 \\
$\mathrm{Fe} 5233.0$ & 346 & 2.94 & $0.18-0.15-0.18$ \\
$\mathrm{Fe} 5250.2$ & 62 & 0.12 & $0.03-0.08-0.03$ & \\
$\mathrm{CN} 3882.5$ & 127 & - & $0.02-0.05-0.02$ & 0 \\
$\mathrm{H} \alpha 6562.8$ & - & - & $0.50-0.50-0.50$ & 2000 \\
$\mathrm{H} \beta 4861.3$ & - & - & $0.20-0.165-0.20$ & 2500 \\
$\mathrm{Ca}+\mathrm{K}_{3} 3933.7$ & - & - & $0.12-0.10-0.12$ & 5000 \\
\hline
\end{tabular}
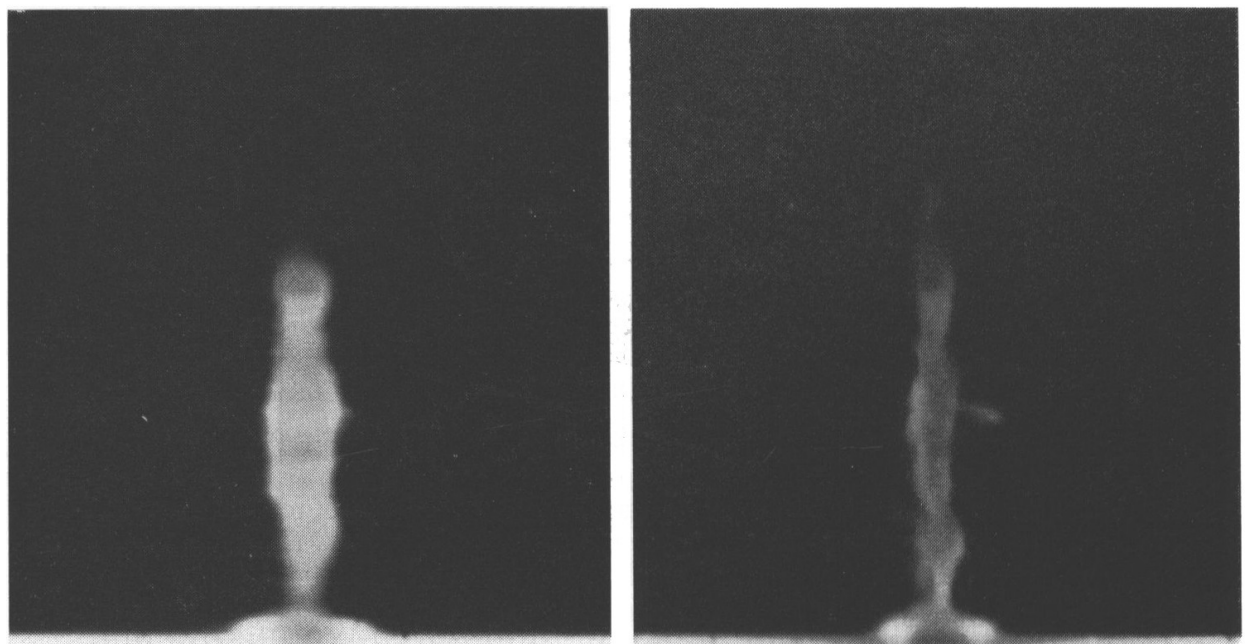

Fig. 3. $\mathrm{H} \alpha$ (left) and $\mathrm{Ca}^{+} \mathrm{K}$ (right) spectra taken with slit radial to limb, showing narrow features that are only visible at $\mathrm{Ca}+\mathrm{K}$.

sensed and so the effective height of formation runs lower than usually quoted for these lines. Thus, because of the slit position, $\mathrm{H} \beta$ is shown originating higher than $\mathrm{H}$. The heights listed are interpolated values taken from De Jager (1959), while other spectroscopic data are based on Moore et al. (1966).

Results for observations in 1968-69 are given in Table III and discussed in Section 4. 
TABLE III

Equatorial angular velocity deg/day, sidereal, derived for height sensitive lines 1968-69

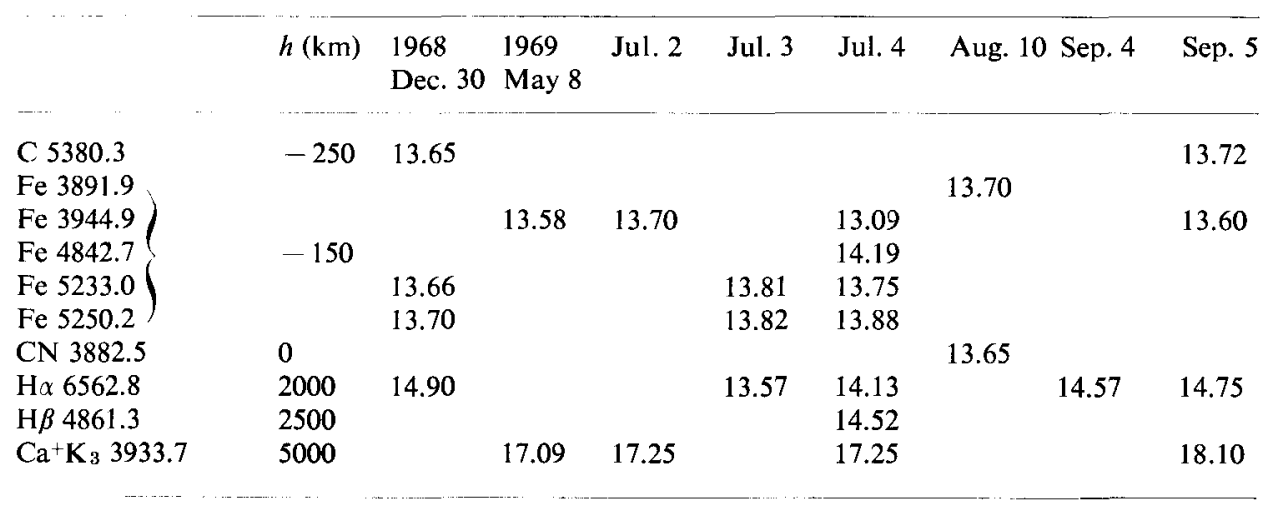

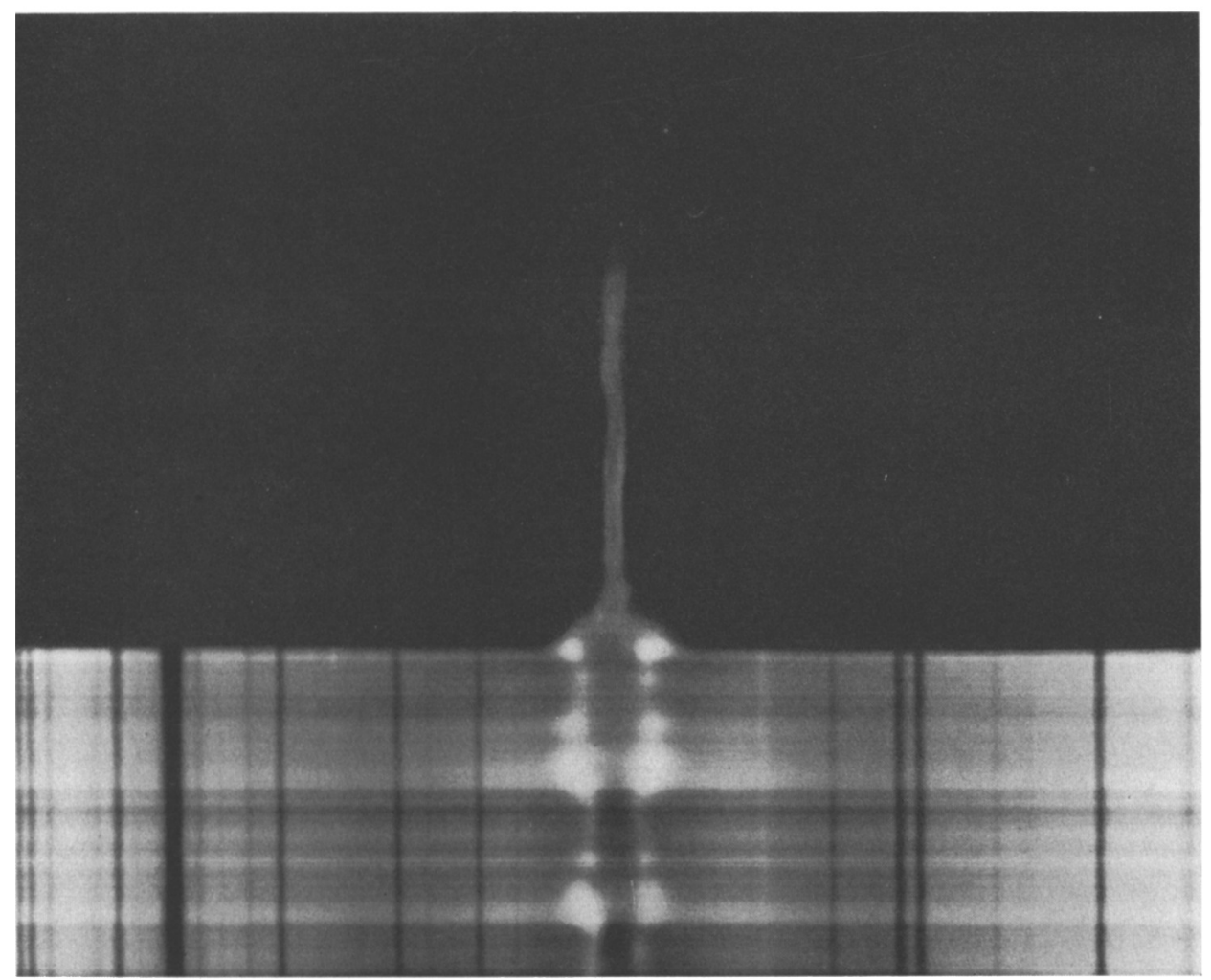

Fig. 4. Quiescent prominence in $\mathrm{Ca}+\mathrm{K}$, radial slit. 


\section{The Prominence Observations}

In an interesting series of papers Evershed $(1927,1929,1935,1945)$ has reported a striking increase in angular velocity with height based on displacements of the $\mathrm{H}$ and $\mathrm{K}$ lines in prominences. Although disputed by others (cf. Perepelkin, 1932) the conflicting observations have generally been made at comparatively low dispersion or using $\mathrm{H} \alpha$ (cf. Liszka, 1969). O. Engvold pointed out to the writer that the K-line in certain prominences is very narrow. High dispersion $(0.09 \AA / \mathrm{mm})$ material has shown that frequently a prominence has a broad turbulent part co-mingling with a very narrow central core (Figure 3 ).
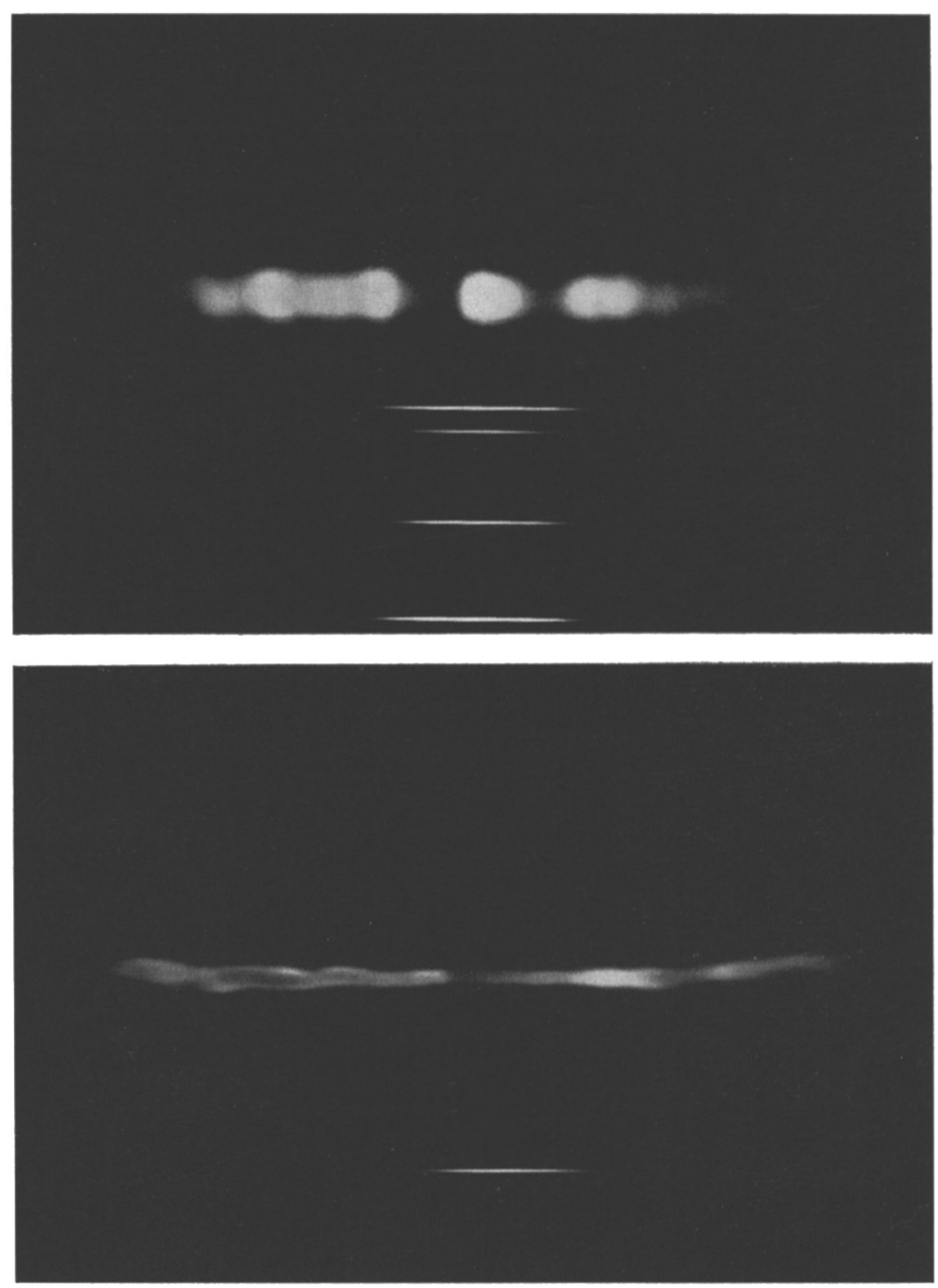

Fig. 5. $\mathrm{H} \alpha$ (top) and $\mathrm{Ca}^{+} \mathrm{K}$ (bottom) spectra of quiescent prominence taken with slit $27000 \mathrm{~km}$ above and tangent to the limb. Comparison spectrum is thorium. 
Often quiescent prominences show extensive regions having the narrow K-line characteristic (Figures 4 and 5). We have recently begun to systematically obtain $\mathrm{K}$-line spectra of prominences and in the reduction of these plates for solar rotation special weight is given to the narrow line measurements. At the time of writing (Sept. 1969) 60 narrow line objects have been measured by Mr. Daniel Gezari, but more material is needed before the results of Evershed can be confirmed.

\section{Discussion of Results for the Disk}

An inspection of Table III discloses two trends. First the rotation rates vary with date. This variable characteristic is further amplified by our previous results extending three years back (Livingston, 1969a). We presume this scatter is mainly a consequence of our inability to average-out the long-lived and large scale convective patterns discussed in the first section.

Second, an increasing angular velocity with height is noticed. The chromospheric lines taken as a whole show almost a deg/day, or a $7 \%$, increase over the low lying photosphere. No certain variation is detected within the photosphere (see also Livingston, 1969b). The velocity obtained for $\mathrm{Ca}^{+} \mathrm{K}_{3}$ is particularly large, but then this line may be formed appreciably higher than $\mathrm{H} \alpha$ and $\mathrm{H} \beta$.

A summary of the observed rotation with height within the solar envelope is given in Figure 6. Aslanov (1963) found a wide range of velocity within the photosphere, but this is yet to be confirmed. Our own findings are not new but rather parallel the discovery of Adams (1911) who first showed the increase of velocity with height as so indicated. Finally the $1967 \mathrm{~K}$-corona tracer (coronal condensations) measurements of Hansen et al. (1969) indicate a possible retardation of velocity in the intermediate

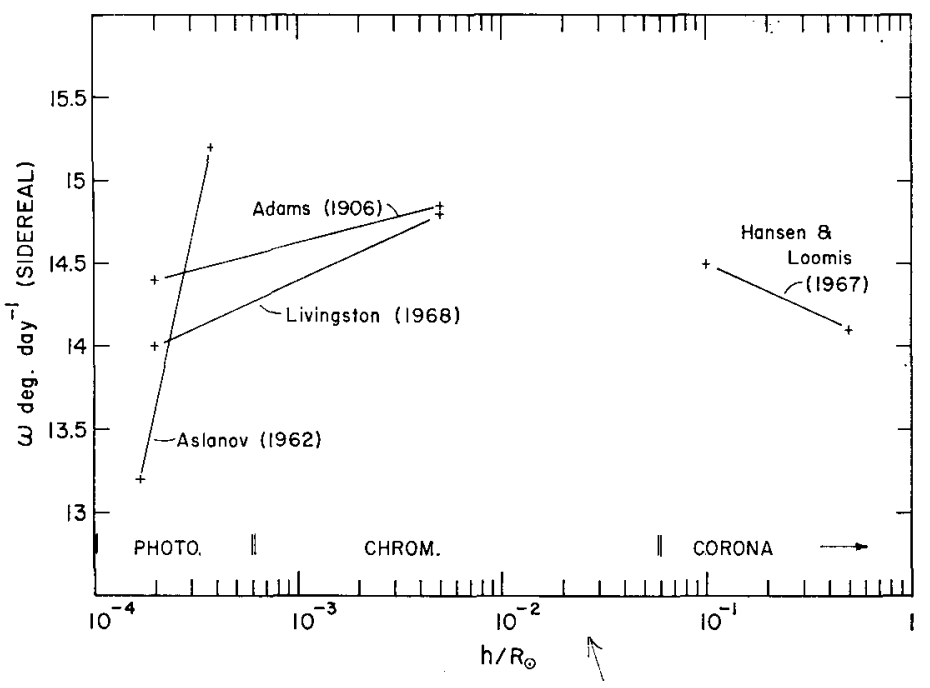

Fig. 6. Summary of the observational data of variation of angular rotation with height. See text . 
corona. One concludes from these data that there is an increase of velocity with height through the chromosphere of up to about $7 \%\left(30 \%\right.$ if the $\mathrm{K}_{3}$ data prove correct) and then a subsequent fall off through the outer corona.

What are the stellar consequences of these observations? At this Colloquium we have heard a number of theories discussed wherein momentum is transferred outward from the star's surface to a stellar wind, with a resulting braking action being exerted on the star's rotation. Brandt (1966) has similarly considered this effect on the sun. If, however, the angular velocity increases outward the reverse would seem to be the case, i.e. momentum would flow downward. The detailed mechanism for creating these conditions has, of course, yet to be given. The latitude dependence of this height gradient, not given here, may prove crucial.

\section{References}

Adams, W. S.: 1911, Carnegie Inst. of Washington Publ., No. 138.

Aslanov, I. A.: 1963, Astron. Zh. 40, 1036 (Soviet Astron. - AJ 7, 794).

Brandt, J. C.: 1966, Astrophys. J. 144, 1221.

De Jager, C.: 1959, Handbuch der Physik (ed. by S. Flügge), Springer-Verlag, Berlin.

Evershed, J.: 1927, Monthly Notices Roy. Astron. Soc. 88, 126.

Evershed, J.: 1929, Monthly Notices Roy. Astron. Soc. 89, 250.

Evershed, J.: 1935, Monthly Notices Roy. Astron. Soc. 95, 503.

Evershed, J.: 1945, Monthly Notices Roy. Astron. Soc. 105, 204.

Hansen, R. T., Hansen, S. F., and Loomis, H. G.: 1969, Solar Phys., in press.

Liszka, L.: 1969, Kiruna Geophys. Obs. Report, No. 691.

Livingston, W. C.: 1969a, Solar Phys. 7, 144.

Livingston, W. C.: 1969b, Solar Phys. (in press).

Moore, C. E., Minnaert, M. G. J., and Houtgast, J.: 1966, N.B.S. Monograph 61.

Perepelkin, E. J.: 1932, Pulkovo Obs. Circ. No. 1.

\section{Discussion}

Dicke: One possible means of increasing the rotational velocity of the chromosphere requires back bombardment by the corona. Angular momentum is transferred from the sun to the corona by magnetic stresses but gas flow along magnetic flux strands to the chromosphere from the corona would transfer angular momentum to the upper chromosphere.

Roxburgh: It need not be as difficult to transport angular momentum from a region of low momentum to one of the high momentum. Non-linear waves could do this at the same time as heat is transported up to the corona. 